\title{
The Prognostic Value of LDH And ADA in Serum and Pleural Fluid of Non-Small Cell Lung Cancer
}

\author{
Shuang Zhao \\ West China Hospital, Department of Respiratory and Critical Care Medicine \\ Jun Zeng ( $\nabla$ tonypersist@126.com ) \\ Chengdu Medical College \\ Juanli Wu \\ West China Hospital, Department of Laboratory Medicine
}

\section{Research}

Keywords: LDH, ADA, cancer ratio, non-small cell lung cancer, prognosis

Posted Date: October 28th, 2021

DOI: https://doi.org/10.21203/rs.3.rs-955211/v1

License: (1) This work is licensed under a Creative Commons Attribution 4.0 International License. Read Full License 


\section{Abstract}

Objective: To explore the value of dehydrogenase (LDH) and adenosine deaminase (ADA) in serum and pleural fluid in predicting the prognosis of non-small cell lung cancer.

Methods: A total of 134 patients with non-small cell lung cancer (NSCLC) with pleural effusion were enrolled. LDH in Serum and LDH and ADA in pleural fluid were detected, and the cancer ratio (serum LDH/pleural fluid ADA ratio) was calculated. Patients were followed until death. To analyze the correlation between the above indicators and the clinical pathological characteristics of the patients, and the predictive value for the prognosis of the patients.

Results: Among the included NSCLC patients with pleural effusion, the average age was 61.76 years old, mainly male (66.7\%), the right lung was more common (47.2\%), adenocarcinoma was the main (82.1\%), and smoking patients accounted for $48.0 \%$. In the diagnosis method, there were 94 cases were diagnosed by pleural fluid exfoliated cells or pleural biopsy. In terms of clinical features, serum LDH is only related to adrenal metastasis $(p=0.000)$, and pleural fluid LDH is related to the patient's pathological type $(p=0.001)$. There was no correlation between pleural fluid ADA or cancer ratio and patient gender, lesion location, pathological type, diagnosis method, smoking history, and metastatic location. In terms of prognostic value, patients with ADA < $20 U / L$ and cancer ratio $>20$ have shorter overall survival and worse prognosis $(p<0.0001$ and $p=0.0178$, respectively). The multivariate Cox regression analysis suggested that the cancer ratio $(H R=1.699,95 \% \mathrm{Cl}: 1.097-2.630, p=0.017)$ was an independent risk factor for the poor prognosis of patients.

Conclusion: The NSCLC patients with pleural fluid ADA $<20 \mathrm{U} / \mathrm{L}$ and cancer ratio $>20$ combined with pleural effusion had a shorter overall survival and worse prognosis.

\section{Introduction}

Lung cancer is one of the leading malignant tumors with the highest morbidity and mortality worldwide, accounting for $11.6 \%$ and $18.4 \%$ of all tumors respectively, and seriously threatens human health ${ }^{[1,2]}$. Regrettably, there is still a lack of effective approach to the prevention and cure of lung cancer. Of which, advanced lung cancer patients with pleural effusion have severely impaired quality of life, high treatment failure rate and poor prognosis. Notably, there are still significant differences in the survival between different individuals of advanced lung cancer patients with pleural effusion. For those patients with different prognosis, adopting more precise therapies may obtain better quality of life and prognosis. However, there is still a lack of clinical indicators that can effectively predict the prognosis of lung cancer patients with pleural effusion. Therefore, this study detected the levels of serum LDH, pleural fluid LDH, pleural fluid ADA, and serum LDH/pleural fluid ADA ratios of non-small cell lung cancer (NSCLC) patients with pleural effusion, and explored the relationship between them and the clinical pathological characteristics and prognosis of patients, and clarified the clinical value of them in predicting poor prognosis in NSCLC patients with malignant pleural effusion.

\section{Objects And Methods}

\subsection{Research objects}

A total of 134 NSCLC patients with malignant pleural effusion diagnosed by pathological biopsy from September 2009 to September 2013 in the Department of Respiratory and Critical Care Medicine, West China Hospital of Sichuan University were enrolled. Inclusion criteria: 1.All patients were diagnosed as NSCLC by pathological biopsy or cytological diagnosis; 2.All patients had not received radiotherapy, chemotherapy, targeted therapy or immunotherapy before diagnosis, and had complete clinical data; 3.Both chest CT and color Doppler ultrasound revealed pleural effusion; 4. Follow-up were completed for all patients; 5. Exclude pulmonary tuberculosis, autoimmune diseases, other malignant tumors, severe heart failure, severe liver and kidney dysfunction, and severe hypoproteinemia. The data collected include age, gender, lesion locations, pathological types, diagnosis methods, smoking history, lymph node metastases, and distant metastases. The patients were followed up regularly until the death of the patients, and the survival time was recorded. The pathological types and differentiation degrees were based on the 1999 WHO classification of lung cancer histological types ${ }^{[3]}$.

Page $2 / 11$ 


\subsection{Methods}

All patients were fasting for over 12 hours until the next morning after admission. $5 \mathrm{ml}$ blood from one side of the elbow vein was drawn and injected into a biochemical tube without anticoagulant. Then the patients underwent thoracentesis under local anesthesia, $10 \mathrm{ml}$ of pleural fluid were drawn and sent to the laboratory for testing immediately. Venous blood and pleural effusion were centrifuged at $3000 \mathrm{r} / \mathrm{min}$ for $10 \mathrm{~min}$ by routine method. After collecting the supernatant, lactate dehydrogenase (LDH) and adenylate aminase (ADA) are detected by Roche automatic biochemical analyzer (cobas 8000 ) via biochemical analysis and chemiluminescence method. LDH detection reagent uses Roche lactate dehydrogenase detection kit (colorimetric Method), and ADA detection reagent adopts Zhongyuan Biological Adenosine Aminase Kit (enzymatic method). All operations are performed in accordance with the instructions. According to previous literature reports and the normal reference range of the instructions, the positive criterion include serum LDH greater than the normal reference range (135-225 U/L), pleural fluid LDH $\geq$ $500 U / L$ and pleural fluid ADA > 20U/L. Cancer Ratio (Cancer Ratio) is serum LDH/pleural effusion ADA, and its ratio > 20 is considered positive.

\subsection{Statistical analysis}

Office Excel 2016 is used for data entry and storage, and measurement data is expressed by mean \pm standard deviation. SPSS 20.0 was used for statistical analysis, and the $\chi 2$ test, $t$ test, rank sum test and Fisher exact probability were used for comparison between groups. Survival analysis were performed by Kaplan-Meier and Log-rank analysis, and the prognostic factors were analyzed by multivariate COX regression model. GraphPad Prism was used for picture production. A two-sided $p<0.05$ denoted that the difference was statistically significant.

\section{Result}

\subsection{General situation}

A total of 123 NSCLC patients with pleural effusion were included in this study. The clinicopathological characteristics are summarized in Table 1. The patients ranged in age from 28 to 85, with an average age of 61.76 years. Among which 82 cases were male (66.7\%) and 41 cases were female (33.3\%). The main lesion was in the right lung, with 58 cases (47.2\%), 53 cases $(43.1 \%)$ in the left lung, and only 9 cases were diffuse and multiple in both lungs. Adenocarcinoma was the main pathological type, with 101 cases (82.1\%), 15 cases (12.2\%) was squamous cell carcinoma, and 7 cases (5.7\%) was other types. Among these patients 69 were smokers (48.0\%). There were 94 cases diagnosed by pleural fluid exfoliated cells or pleural biopsy, which is the primary diagnosis method. Except intrathoracic metastasis, lymph node metastasis was the most common site (74 cases), followed by bone metastasis ( 23 cases). In terms of overall survival, $56.9 \%$ of patients survived less than 1 year, $32.5 \%$ survived for 1 to 2 years, $7.3 \%$ survived for 2 to 3 years, and only 3.3\% survived more than 3 years.

\subsection{Correlation analysis of LDH, ADA and the clinical characteristics of NSCLC patients with pleural effusion}

In this study, the correlation between LDH, ADA or cancer ratio and patient gender, lesion location, pathological type, diagnosis method, smoking history, metastasis location, and survival were investigated. The results are shown in Table 2. Serum LDH is only related to adrenal metastasis $(p=0.000)$. While pleural fluid LDH is related to the patient's pathological type $(p=0.001)$, among which the level of pleural fluid LDH in adenocarcinoma is the lowest $(430.7 \pm 338.3 \mathrm{U} / \mathrm{L})$, and the level of pleural fluid LDH in squamous cell carcinoma is the highest $(1592.9 \pm 3116 \mathrm{U} / \mathrm{L})$. However, there is no correlation between pleural fluid ADA, cancer ratio and patient gender, lesion location, pathological type, diagnosis method, smoking history, and metastatic location ( $p$ $>0.05$ ). In terms of survival time, ADA and cancer ratio show a contrary tendency, the higher the ADA, the longer the patient's survival time, and the lower the cancer ratio, the longer the patient's survival time; however, the difference is not statistically significant ( $p=0.072$ and $p=0.083$, respectively). 


\subsection{The prognostic value of LDH and ADA in predicting the prognosis of NSCLC patients with pleural effusion}

In this study, the included patients were followed up until the death of the patient. According to the different levels of LDH, ADA and cancer ratio, they are divided into positive group and negative group, and further analyze the above indicators and the prognosis of patients. In univariate analysis, serum and pleural fluid LDH expression levels were not correlated with the prognosis of NSCLC patients with pleural effusion ( $p=0.2988$, as shown in Figure $1 \mathrm{~A}$ and $p=0.0752$, as shown in Figure 1B, respectively). Patients with reduced ADA in pleural fluid $(<20 \mathrm{U} / \mathrm{L})$ have a shorter overall survival and a worse prognosis $(p<0.0001$, Figure $1 C)$. The prognosis of patients with high cancer ratio $(\geq 20 \mathrm{U} / \mathrm{L})$ is also worse $(p=0.0178$, Figure 1D).

The multivariate Cox regression model was further used to analyze the impact of various indicators on the prognosis of patients, the results are shown in Table 3. The cancer ratio is an independent prognostic factor for NSCLC patients with pleural effusion. For which the median survival of patients with cancer ratio $\geq 20 \mathrm{U} / \mathrm{L}$ was 8 months, and 11.5 months for patients with $<20 \mathrm{U} / \mathrm{L}$ $(\mathrm{HR}=1.699,95 \% \mathrm{Cl}: 1.097-2.630, \mathrm{p}=0.017)$. However, the multivariate analysis of serum LDH, pleural fluid LDH, pleural fluid ADA and prognosis were not statistically different $(p>0.05)$.

\section{Discussion}

This study explores the prognostic value of serum and pleural fluid LDH and ADA expression in NSCLC patients with pleural effusion. The results show that serum LDH is related to adrenal metastasis, while pleural fluid LDH is related to the patient's pathological type in terms of clinical features. For the prognostic predicting value, the overall survival of patients with ADA < $20 U / L$ and cancer ratio $>20$ is shorter and its prognosis is worse. However, neither serum LDH nor pleural fluid LDH is related to the prognosis of patients. In clinical practice, serum and pleural fluid LDH and ADA expression level are easy to be obtained at different levels of hospitals. This study especially indicates that the cancer ratio has an important predictive value for the prognosis of advanced NSCLC patients with pleural effusion, which can provide a basis for better predicting the prognosis of patients in the clinic, and for individualized treatment in advance and follow-up.

LDH is the main enzyme in carbohydrate metabolism, which exist in normal serum. When tumor cells proliferate actively and have amount of necrosis, the enzymes in tumor tissues can be released into the blood, resulting in the increased activity of serum LDH. It is reported that LDH concentration is significantly associated with an increase in the total mortality of lung cancer patients $(H R=1.49,95 \% \mathrm{Cl}, 1.38-1.59)$, and the subgroup analysis of the study also showed that high levels of low-density lipoprotein was associated with the significant increase in mortality of small cell lung cancer (SCLC) patients $(\mathrm{HR}=1.54,95 \% \mathrm{Cl}$, 1.43-1.67) or NSCLC patients $(\mathrm{HR}=1.25,95 \% \mathrm{Cl}: 1.06-1.46){ }^{[4]}$. Another study also showed that the hazard ratio $(\mathrm{HR}=1.45,95 \%$ Cl: 1.27 1.66) of the overall survival rate of SCLC patients with elevated serum LDH was increased ${ }^{[5]}$. And LDH > 1500 U/L in pleural fluid predicts that the patient will survive less than 1 year ${ }^{[6]}$. However, our study only suggests that serum LDH is related to adrenal metastasis, and pleural fluid LDH is related to the patient's pathological type; it does not suggest that serum or pleural fluid LDH elevation is associated with the prognosis of advanced NSCLC patients with pleural effusion. The inconsistent results may be due to differences in the study population. This study only included NSCLC patients with pleural effusion; on the other hand, it may be related to the inconsistent cut-off value of increased LDH. Therefore, the study of LDH in lung cancer patients needs to be more refined, and more studies are needed for further verification.

ADA is an important enzyme widely distributed in the human body, especially in T lymphocytes. It participates in the catabolism of purine nucleosides and can specifically catalyze the hydrolysis of nucleosides to generate inosine and ammonia. ADA increased significantly in tuberculous pleural effusion with type IV allergies. The sensitivity and specificity for the diagnosis of tuberculous pleural effusion or empyema via the LDH/ADA ratio greater than 15.5 can reach $62.0 \%$ and $91.0 \%$, respectively ${ }^{[7]}$. In cancer patients, lymphocyte ADA activity is reduced ${ }^{[8]}$, and it is related to the advanced stage of the patient. In the study of malignant pleural effusion, compared with normal ADA, low and high ADA levels $(<15, \geq 40)$ are associated with worse survival rates for lymphoma, lung cancer or breast cancer $(p=0.0024)^{[9]}$. This study shows that the overall survival of NSCLC patients with pleural effusion and $A D A<20 \mathrm{U} / \mathrm{L}$ is shorter and the prognosis is worse. Therefore, ADA has important value in 
distinguishing malignant pleural effusion from benign one and predicting the prognosis of patients with malignant pleural effusion.

The cancer ratio is the ratio of serum LDH to pleural fluid ADA. Akash Verma et al. conducted a study on 163 patients with pleural effusion of different causes, and the results showed that ADA-negative pleural fluid was associated with malignant tumors (HR = $062,95 \% \mathrm{Cl}: 0.45-0.85, \mathrm{p}=0.003)$, while serum lactate dehydrogenase $(\mathrm{HR}=1.02,95 \% \mathrm{Cl}: 1.0-1.03, \mathrm{p}=0.004)$ and cancer ratio $(\mathrm{HR}=0.94,95 \% \mathrm{Cl}: 0.99-1.0, \mathrm{p}=0.04)$ were positively correlated with malignant pleural effusion. Cancer ratio $>20$ can be used to distinguish benign and malignant pleural effusions, with sensitivity and specificity of 0.95 and 0.85 respectively ${ }^{[10]}$. The team further carried out a prospective study on the value of cancer ratio. A total of 118 patients were enrolled, including 84 cases of malignant pleural effusion and 34 cases of tuberculosis. The results showed that the sensitivity and specificity of malignant pleural effusion determined by the cancer ratio $>20$ were 0.95 . (95\% Cl: 0.87-0.98) and 0.85 (95\% Cl: 0.68-0.94) respectively [10] our study further evaluated the relationship between cancer ratio and patient prognosis in NSCLC patients with pleural effusion, and showed that patients with cancer ratio $>20$ had shorter overall survival and worse prognosis. Therefore, the increased cancer ratio can distinguish benign and malignant pleural effusions, and can predict the poor prognosis of patients with advanced NSCLC. However, the above conclusions require larger sample and prospective studies to verify.

In summary, the results of this study found that NSCLC patients with pleural effusion combined with ADA $<20 \mathrm{U} / \mathrm{L}$ and cancer ratio $>20$ have a shorter overall survival period and a worse prognosis. This study can provide a basis for better predicting the prognosis of patients in clinical practice, and for individualized treatment in advance and follow-up.

\section{Declarations}

\section{Consent for publication:}

Not applicable.

\section{Availability of data and materials:}

The software, databases and all relevant raw data are freely available to scientists.

\section{Competing interests:}

The authors declare that they have no conflict of interest.

\section{Funding:}

This work was supported by the Applied Basic Research of Sichuan Department of Science and Technology (2021YJ0470), the Youth Innovation Project of Sichuan Medical Association (Q17025).

\section{Author contributions:}

JZ and SZ designed and performed experiments, JLW and SZ collected the clinical samples and patients' information, and SZ analyzed data and wrote the manuscript.

\section{Acknowledgements[}

None.

\section{References}

[1] F. Bray, J. Ferlay, I. Soerjomataram, R. L. Siegel, L. A. Torre, A. Jemal. Global cancer statistics 2018: GLOBOCAN estimates of incidence and mortality worldwide for 36 cancers in 185 countries. CA: a cancer journal for clinicians[J]. 2018, 68(6): $394-424$. 
[2] K. X. Sun, R. S. Zheng, H. M. Zeng, S. W. Zhang, X. N. Zou, X. Y. Gu, C. F. Xia, Z. X. Yang, H. Li, W. Q. Chen, J. He. [The incidence and mortality of lung cancer in China, 2014]. Zhonghua zhong liu za zhi [Chinese journal of oncology][J]. 2018, 40(11): 805-811.

[3] W. D. Travis, E. Brambilla, G. J. Riely. New pathologic classification of lung cancer: relevance for clinical practice and clinical trials. Journal of clinical oncology : official journal of the American Society of Clinical Oncology[J]. 2013, 31(8): 992-1001.

[4] T. Deng, J. Zhang, Y. Meng, Y. Zhou, W. Li. Higher pretreatment lactate dehydrogenase concentration predicts worse overall survival in patients with lung cancer. Medicine[J]. 2018, 97(38): e12524.

[5] X. Zhang, M. Guo, J. Fan, Z. Lv, Q. Huang, J. Han, F. Wu, G. Hu, J. Xu, Y. Jin. Prognostic significance of serum LDH in small cell lung cancer: A systematic review with meta-analysis. Cancer biomarkers : section A of Disease markers[J]. 2016, 16(3): 415-23.

[6] A. Verma, C. K. Phua, W. Y. Sim, R. E. Algoso, K. S. Tee, S. J. Lew, A. Y. Lim, S. K. Goh, D. Y. Tai, A. C. Kor, B. Ho, J.

Abisheganaden. Pleural LDH as a prognostic marker in adenocarcinoma lung with malignant pleural effusion. Medicine[J]. 2016, 95(26): e3996.

[7] T. Saraya, K. Ohkuma, T. Koide, H. Goto, H. Takizawa, R. W. Light. A novel diagnostic method for distinguishing parapneumonic effusion and empyema from other diseases by using the pleural lactate dehydrogenase to adenosine deaminase ratio and carcinoembryonic antigen levels. Medicine[J]. 2019, 98(13): e15003.

[8] D. Zanini, L. H. Manfredi, L. P. Pelinson, V. C. Pimentel, A. M. Cardoso, V. D. Carmo Araújo Gonçalves, C. B. D. Santos, J. M. Gutierres, V. M. Morsch, D. B. R. Leal, M. R. C. Schetinger. ADA activity is decreased in lymphocytes from patients with advanced stage of lung cancer. Medical oncology (Northwood, London, England)[J]. 2019, 36(9): 78.

[9] R. M. Terra, L. Antonangelo, A. W. Mariani, R. L. de Oliveira, L. R. Teixeira, P. M. Pego-Fernandes. Pleural Fluid Adenosine Deaminase (ADA) Predicts Survival in Patients with Malignant Pleural Effusion. Lung[J]. 2016, 194(4): 681-6.

[10] A. Verma, R. S. Dagaonkar, D. Marshall, J. Abisheganaden, R. W. Light. Differentiating Malignant from Tubercular Pleural Effusion by Cancer Ratio Plus (Cancer Ratio: Pleural Lymphocyte Count). Canadian respiratory journal[J]. 2016, 20167348239.

\section{Tables}

Table 1. Clinical characteristics of NSCLC patients with pleural effusion $(\mathrm{N}=123)$. 


\begin{tabular}{|c|c|}
\hline Variables & Results \\
\hline Age, mean (SD) & $61.76(28-85)$ \\
\hline \multicolumn{2}{|l|}{ Gender } \\
\hline Male, n (\%) & $82(66.7 \%)$ \\
\hline Female, n (\%) & $41(33.3 \%)$ \\
\hline \multicolumn{2}{|l|}{ Lesion location } \\
\hline Left lung, n (\%) & $53(43.1 \%)$ \\
\hline Right lung, $n(\%)$ & $58(47.2 \%)$ \\
\hline Diffuse, n (\%) & $9(7.3 \%)$ \\
\hline Others, n (\%) & $3(2.4 \%)$ \\
\hline \multicolumn{2}{|l|}{ Pathologic types } \\
\hline Adenocarcinoma, n (\%) & $101(82.1 \%)$ \\
\hline Squamous carcinoma, $\mathrm{n}(\%)$ & $15(12.2 \%)$ \\
\hline Others, $\mathrm{n}(\%)$ & $7(5.7 \%)$ \\
\hline \multicolumn{2}{|l|}{ Diagnosis methods } \\
\hline Operation, $\mathrm{n}$ & 6 \\
\hline Bronchofibroscope, $n$ & 29 \\
\hline Percutaneous lung biopsy, $\mathrm{n}$ & 11 \\
\hline Exfoliated cell in sputum, $n$ & 10 \\
\hline Pleural biopsy or exfoliated cells in pleural effusion, $n$ & 94 \\
\hline Lymph node biopsy, $n$ & 2 \\
\hline \multicolumn{2}{|l|}{ Smoking history } \\
\hline No, $n(\%)$ & $64(52.0 \%)$ \\
\hline yes, n (\%) & $59(48.0 \%)$ \\
\hline \multicolumn{2}{|l|}{ Other metastases } \\
\hline Lymph, n (\%) & 74 \\
\hline Lung, n (\%) & 8 \\
\hline Brain, n (\%) & 8 \\
\hline Bone, n (\%) & 23 \\
\hline Liver, n (\%) & 8 \\
\hline Adrenal gland, $\mathrm{n}(\%)$ & 8 \\
\hline \multicolumn{2}{|l|}{ Survival time } \\
\hline 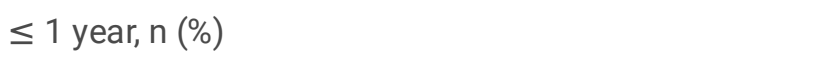 & $70(56.9 \%)$ \\
\hline 1 to 2 years, $n(\%)$ & $40(32.5 \%)$ \\
\hline 2 to 3 years, $n(\%)$ & $9(7.3 \%)$ \\
\hline$\geq 3$ years, $n(\%)$ & $4(3.3 \%)$ \\
\hline
\end{tabular}

Page $7 / 11$ 


\begin{tabular}{|lc|}
\hline Therapies & \\
\hline Radiotherapy, $\mathrm{n}$ & 19 \\
\hline Chemotherpy, $\mathrm{n}$ & 61 \\
\hline Operation, $\mathrm{n}$ & 18 \\
\hline Target therapy, $\mathrm{n}$ & 23 \\
\hline Others, $\mathrm{n}$ & 21 \\
\hline
\end{tabular}

Table 2. Correlation analysis between LDH, ADA and the clinicopathologic features of NSCLC patients with pleural effusion. 


\begin{tabular}{|c|c|c|c|c|c|c|c|c|c|}
\hline Features & $\mathbf{N}$ & Serum LDH & $\mathbf{P}$ & $\begin{array}{l}\text { Pleural } \\
\text { effusion LDH }\end{array}$ & $\mathbf{P}$ & $\begin{array}{l}\text { Pleural } \\
\text { effusion } \\
\text { ADA }\end{array}$ & $\mathbf{P}$ & $\begin{array}{l}\text { Cancer } \\
\text { rato }\end{array}$ & $\mathbf{P}$ \\
\hline Gender & & & 0.879 & & 0.208 & & 0.491 & & 0.363 \\
\hline male & 72 & $213.9 \pm 97.4$ & & $678.6 \pm 1407$ & & $15.5 \pm 20.7$ & & $21.9 \pm 14.2$ & \\
\hline female & 41 & $217.4 \pm 1401$ & & $396.1 \pm 335.2$ & & $18.8 \pm 30.6$ & & $24.5 \pm 16.1$ & \\
\hline Lesion location & & & 0.997 & & 0.432 & & 0.441 & & 0.111 \\
\hline Left lung & 53 & $214.2 \pm 1233$ & & $399.8 \pm 414.0$ & & $20.5 \pm 31.6$ & & $21.4 \pm 12.7$ & \\
\hline Right lung & 58 & $215.4 \pm 106.9$ & & $764.8 \pm 1628$ & & $14.3 \pm 17.7$ & & $29.1 \pm 13.4$ & \\
\hline Diffuse & 9 & $213.7 \pm 69.9$ & & $485.0 \pm 483.6$ & & $10.8 \pm 10.3$ & & $29.0 \pm 13.4$ & \\
\hline Others & 3 & $229.00 \pm 82.4$ & & $656.3 \pm 806.6$ & & $8.50 \pm 4.0$ & & $39.5 \pm 39.4$ & \\
\hline Pathologic types & & & 0.207 & & $0.001^{*}$ & & 0.546 & & 0.318 \\
\hline Adenocarcinoma & 101 & $209.7 \pm 118$ & & $430.7 \pm 338.3$ & & $22.0 \pm 34.1$ & & $21.9 \pm 13.6$ & \\
\hline $\begin{array}{l}\text { Squamous } \\
\text { carcinoma }\end{array}$ & 15 & $216.1 \pm 87.8$ & & $1592.9 \pm 3116$ & & $16.3 \pm 23.5$ & & $25.3 \pm 21.7$ & \\
\hline Others & 7 & $291.4 \pm 152.3$ & & $640.8 \pm 577.7$ & & $10.3 \pm 3.9$ & & $29.7 \pm 13.1$ & \\
\hline \multicolumn{10}{|l|}{ Diagnosis methods } \\
\hline Operation & 6 & $220.3 \pm 98.8$ & 0.912 & $1143.6 \pm 1014$ & 0.232 & $18.5 \pm 6.1$ & 0.847 & $14.4 \pm 11.3$ & 0.160 \\
\hline Bronchofibroscope & 29 & $209.9 \pm 91.7$ & 0.786 & $522.3 \pm 873.9$ & 0.745 & $11.3 \pm 6.2$ & 0.185 & $22.6 \pm 11.2$ & 0.975 \\
\hline $\begin{array}{l}\text { Percutaneous } \\
\text { lung biopsy }\end{array}$ & 11 & $181.1 \pm 73.87$ & 0.317 & $363.5 \pm 158.4$ & 0.514 & $23.9 \pm 14.9$ & 0.367 & $20.7 \pm 14.1$ & 0.644 \\
\hline $\begin{array}{l}\text { Exfoliated cell in } \\
\text { sputum }\end{array}$ & 10 & $262.6 \pm 110.8$ & 0.184 & $384.5 \pm 247.1$ & 0.575 & $9.05 \pm 3.2$ & 0.306 & $34.3 \pm 21.7$ & 0.010 * \\
\hline $\begin{array}{l}\text { Pleural biopsy or } \\
\text { exfoliated cells in } \\
\text { pleural effusion }\end{array}$ & 94 & $216.9 \pm 123.4$ & 0.757 & $549.7 \pm 1221$ & 0.556 & $17.6 \pm 27.5$ & 0.407 & $22.9 \pm 14.1$ & 0.794 \\
\hline $\begin{array}{l}\text { Lymph node } \\
\text { biopsy }\end{array}$ & 2 & $276.5 \pm 231.2$ & 0.459 & $443.0 \pm 357.7$ & 0.864 & $13.4 \pm 3.4$ & 0.853 & $23.5 \pm 23.2$ & 0.940 \\
\hline Smoking history & & & 0.631 & & 0.574 & & 0.855 & & 0.485 \\
\hline No & 64 & $210.2 \pm 130.7$ & & $641.7 \pm 1471$ & & $17.0 \pm 24.8$ & & $21.9 \pm 14.5$ & \\
\hline yes & 59 & $220.4 \pm 102.5$ & & $522.3 \pm 721.1$ & & $16.2 \pm 24.0$ & & $23.7 \pm 15.3$ & \\
\hline \multicolumn{10}{|l|}{ Other metastases } \\
\hline Lymph & 74 & $210.4 \pm 144.2$ & 0.721 & $605.4 \pm 1402$ & 0.808 & $16.3 \pm 23.6$ & 0.868 & $22.8 \pm 13.3$ & 0.938 \\
\hline Lung & 8 & $215.8 \pm 52.5$ & 0.975 & $276.7 \pm 124.4$ & 0.444 & $9.1 \pm 5.1$ & 0.368 & $29.4 \pm 13.7$ & 0.191 \\
\hline Brain & 8 & $221.3 \pm 134.8$ & 0.877 & $283.7 \pm 131.1$ & 0.455 & $13.3 \pm 12.5$ & 0.690 & $20.9 \pm 12.1$ & 0.726 \\
\hline Bone & 23 & $210.7 \pm 98.1$ & 0.397 & $607.2 \pm 957.7$ & 0.918 & $25.5 \pm 37.2$ & 0.054 & $19.7 \pm 13.8$ & 0.272 \\
\hline Liver & 8 & $249.3 \pm 135.4$ & 0.397 & $468.7 \pm 558.2$ & 0.774 & $13.1 \pm 6.5$ & 0.679 & $24.5 \pm 17.9$ & 0.730 \\
\hline Adrenal gland & 8 & $359.8 \pm 270.6$ & $0.000 *$ & $750.1 \pm 621.8$ & 0.681 & $14.6 \pm 9.7$ & 0.807 & $30.1 \pm 18.9$ & 0.150 \\
\hline Survival time & & & 0.130 & & 0.684 & & 0.072 & & 0.083 \\
\hline
\end{tabular}




\begin{tabular}{|c|c|c|c|c|c|c|c|c|c|}
\hline$\leq 1$ year & 70 & $236.6 \pm 135.8$ & & $579.5 \pm 1402$ & & $12.7 \pm 16.1$ & & $25.0 \pm 15.0$ & \\
\hline 1 to 2 years & 40 & $183.7 \pm 85.1$ & & $488.1 \pm 501.2$ & & $21.9 \pm 34.6$ & & $20.7 \pm 14.1$ & \\
\hline 2 to 3 years & 9 & $203.9 \pm 77.4$ & & $1015 \pm 1509$ & & $14.1 \pm 8.7$ & & $21.5 \pm 15.6$ & \\
\hline$\geq 3$ years & 4 & $179 \pm 49.7$ & & $664.2 \pm 648.7$ & & $38.4 \pm 32.5$ & & $7.5 \pm 4.5$ & \\
\hline \multicolumn{10}{|l|}{ Therapies } \\
\hline Radiotherapy & 19 & $223.9 \pm 98.5$ & 0.724 & $543.4 \pm 687.9$ & 0.869 & $14.1 \pm 18.1$ & 0.630 & $27.4 \pm 15.9$ & 0.140 \\
\hline Chemotherpy & 61 & $221.9 \pm 124.8$ & 0.531 & $673.4 \pm 524.2$ & 0.405 & $13.5 \pm 11.1$ & 0.157 & $21.7 \pm 12.3$ & 0.447 \\
\hline Operation & 18 & $223.3 \pm 100.7$ & 0.750 & $674.8 \pm 778.6$ & 0.724 & $12.9 \pm 7.4$ & 0.492 & $26.6 \pm 21.6$ & 0.231 \\
\hline Target therapy & 23 & $214.9 \pm 85.9$ & 0.994 & $529.0 \pm 395.3$ & 0.802 & $13.1 \pm 5.2$ & 0.444 & $19.1 \pm 10.4$ & 0.186 \\
\hline Others & 21 & $190.8 \pm 70.2$ & 0.300 & $982.4 \pm 498.9$ & 0.087 & $11.2 \pm 4.9$ & 0.266 & $20.5 \pm 11.6$ & 0.447 \\
\hline
\end{tabular}

Table 3. The multivariate analysis of the prognostic biomarkers in NSCLC patients with pleural effusion.

\begin{tabular}{|c|c|c|c|c|}
\hline Prognostic biomarkers & Median survival time (month) & HR & $95 \% \mathrm{Cl}$ & $\mathbf{P}$ \\
\hline 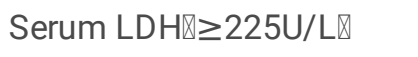 & 7 vs 11 & 0.996 & $0.626-1.586$ & 0.987 \\
\hline $\begin{array}{l}\text { Pleural effusion LDH } \\
(\geq 500 \mathrm{U} / \mathrm{L})\end{array}$ & 12 vs 9 & 1.042 & $0.654-1.659$ & 0.862 \\
\hline 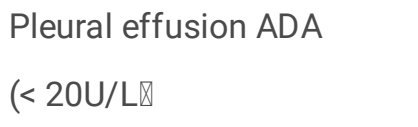 & 9 vs 19 & 0.587 & $0.325-1.059$ & 0.077 \\
\hline Cancer Ratio ( $\geq 20 \rrbracket$ & 8 vs 11.5 & 1.662 & $1.066-2.592$ & $0.025^{\star}$ \\
\hline Chemotherapy (no vs yes) & 11.5 vs 12.7 & 0.963 & $0.664-1.396$ & 0.841 \\
\hline Target therapy (no vs yes) & 8.8 vs 12.9 & 2.371 & $1.428-3.935$ & $0.001 *$ \\
\hline
\end{tabular}

$\mathrm{HR}$, hazard ratio; $\mathrm{Cl}$, confidence interval; ${ }^{*}$, the difference was statistically significant.

\section{Figures}



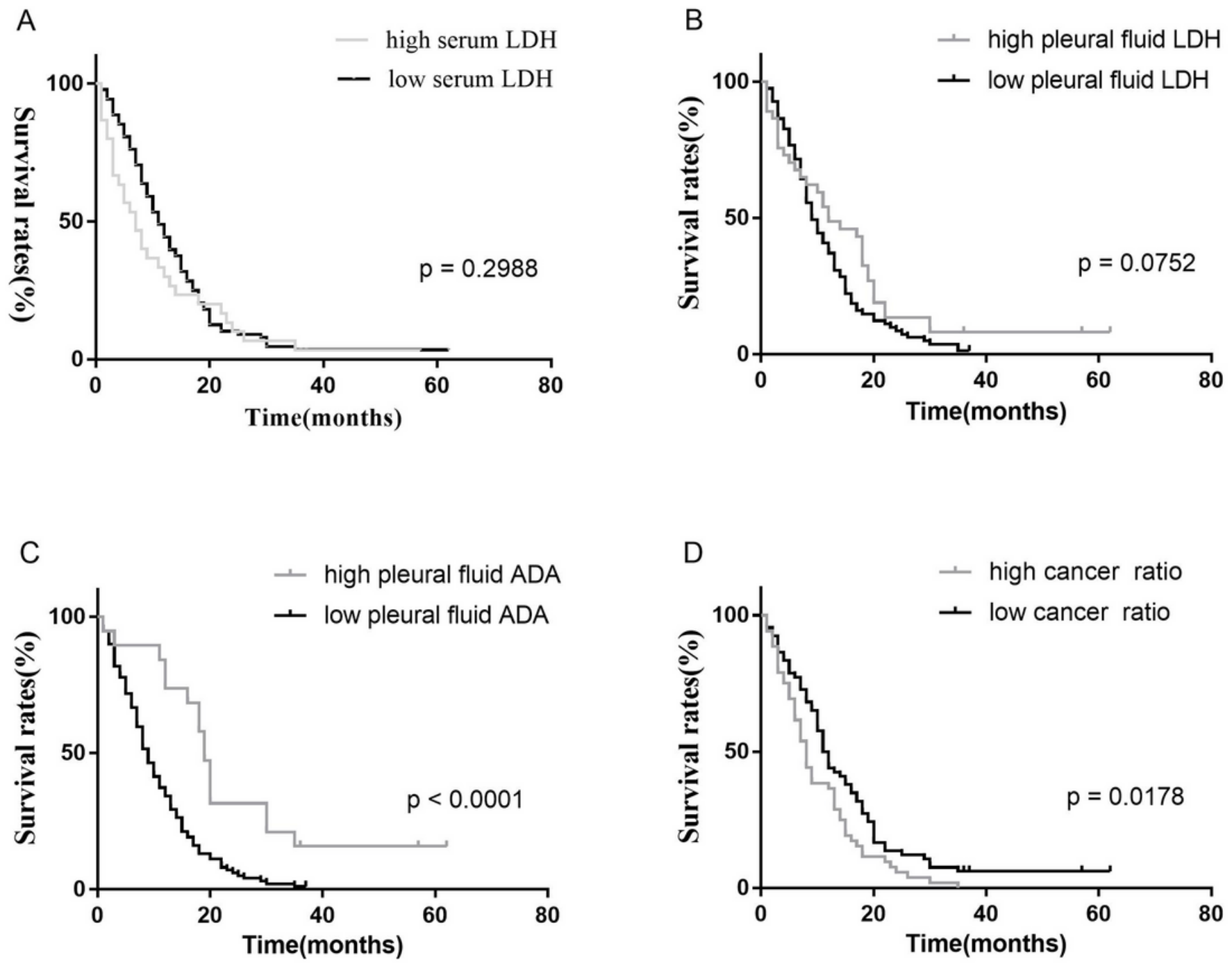

Figure 1

Relationship between serum LDH, pleural fluid LDH, pleural fluid ADA, cancer ratio and prognosis of NSCLC patients with pleural effusion. 